\title{
Metabolomics society 2015 Metabolomics publication awards
}

\author{
Royston Goodacre ${ }^{1}$
}

(C) Springer Science+Business Media New York 2015

In 2013, the Board of the Directors of the Metabolomics Society commissioned a new award scheme to recognise the excellent research published in the journal Metabolomics. Each year, the Society will recognise the top papers within the following categories:

- Best paper award-for the paper with the highest total number of citations during the previous three years. The first author of the winning paper receives a gift certificate from Springer, a certificate from the President of the Society, a front cover image in the Metabolomics journal, and the paper will be made open access for 30 days. The first author of the runnerup paper receives a certificate from the President of the Society.

- Highest download award-for the paper with the highest total number of downloads during the previous three years. The first authors of the winning and runnerup papers each receive a certificate from the President of the Society.

Only primary research papers and "best practice" papers are eligible for these publication awards, which will be announced annually at the international Metabolomics Society conference, typically held in June. Congratulations to the following 2015 winners!

Royston Goodacre

roy.goodacre@manchester.ac.uk

1 School of Chemistry, University of Manchester, Manchester, UK

\section{Best paper award}

- Winner-Szymańska, E., Saccenti, E., Smilde, A.K. \& Westerhuis, J.A. (2012) Double-check: Validation of diagnostic statistics for PLS-DA models in metabolomics studies. Metabolomics 8, S3-S16. http://link. springer.com/article/10.1007/s11306-011-0330-3, doi:10. 1007/s11306-011-0330-3.

- Runner up-Leichtle, A.B., Nuoffer, J-M., Ceglarek, U., Kase, J., Conrad, T., Witzigmann, H., Thiery, J. \& Fiedler, G.M. (2012) Serum amino acid profiles and their alterations in colorectal cancer. Metabolomics $\mathbf{8}$, 643-653. http://link.springer.com/article/10.1007/s11306011-0357-5, doi:10.1007/s11306-011-0357-5.

\section{Highest download award}

- Winner-Cantoria, M.J., Boros, L.G. \& Meuillet, E.J. (2014) Contextual inhibition of fatty acid synthesis by metformin involves glucose-derived acetyl-CoA and cholesterol in pancreatic tumor cells. Metabolomics 10, 91-104. http://link.springer.com/article/10.1007/s11306013-0555-4, doi:10.1007/S11306-013-0555-4.

- Runner up-Cao, B., Li, M., Zha, W., Zhao, Q., Gu, R., Liu, L., Shi, J., Zhou, J., Zhou, F., Wu, X., Wu, Z., Wang, G. \& Aa, J. (2013) Metabolomic approach to evaluating adriamycin pharmacodynamics and resistance in breast cancer cells. Metabolomics 9, 960-973. http://link.springer.com/article/10.1007/s11306-013-05 17-x, doi:10.1007/S11306-013-0517-X. 